\title{
Local Moments in an Interacting Environment
}

\author{
P. Coleman * and A. M. Tsvelik \\ Department of Physics, Oxford University 1 Keble Road Oxford OX2 3NP, UK
}

(August 26, 2021)

\begin{abstract}
We discuss how local moment physics is modified by the presence of interactions in the conduction sea. Interactions in the conduction sea are shown to open up new symmetry channels for the exchange of spin with localized moments. We illustrate this conclusion in the strong-coupling limit by carrying out a Schrieffer Wolff transformation for a local moment in an interacting electron sea, and show that these corrections become very severe in the approach to a Mott transition.

78.20.Ls, 47.25.Gz, 76.50+b, 72.15.Gd
\end{abstract}

\section{INTRODUCTION}

In recent times, the "quantum chemistry" approach has proven one of the most effective ways to formulate minimal models of strongly correlated electron systems. The corresponding strategy of first solving the physics of a strongly interacting atom or cluster, and later superimposing the inter-site couplings has provided an underlying philosephy for many models of interacting electron systems, 12 andled to several new concepts, such as the "lpcal moment" $\mathrm{t}$, the "upper" and "lower" Hubbard bandst and the "Zhang Rice singlet".

A key underlying assumption of the quantum chemistry approach is that the interacting environment which develops around each local scattering center, atom or cluster, does not qualitatively change its scattering properties. This long-held assumption may not hold in all densely interacting systems and for this reason, deserves special scrutiny. We already know that this assumption fails in one dimension, where interactions in the bulk Luttinger liquid alter the scaling exponents for forward and backward scattering, qualitatively changing the character of the scattering center. A weak potential scatterer renor malizes into an infinitely strong blockade to transport, whilst a one-channel Kondo develops-properties reminiscent of a two-channel Kondo effect. 5

Motivated by these considerations, this paper discusses how an interacting environment can qualitatively modify the scattering properties of a local moment in higher dimensions. In one dimension, forward and backward scattering are delineated by their effects on spin-charge coupling: the former preserves spin-charge decoupling, whereas the latter couples spin and charge together. This accounts for their very different scaling properties in the presence of interactions. In higher dimensions, spin exchange between a local moment and its environment can be similarly divided, and in keeping with the lower dimensional analog, Coulomb interactions tend to suppress those components of the spin scattering that couple to charge currents. Some aspects of these effects have been discussed by Schork and Fulded. Our paper serves to highlight a particular point, namely that this effect gives rise to new spin-exchange channels between the local moment and its environment. In the lattice, these new scattering channels qualitatively modify the interactions between mobile Kondo singlets. A forthcoming paper 10 will discuss how second channel scattering in a Kondo lattice can give rise to a collective Kondo effect that destabilizes the Fermi liquid and ultimately gives rise to composite pairing 11

\section{MAGNETIC IMPURITY IN A NON-INTERACTING ENVIRONMENT}

The usual starting point for studying a magnetic impurity is the Anderson impurity modell. We shall examine how the reduction of the Anderson model to a Kondo model is affected by the presence of interactions amongst the conduction electrons. We begin with a brief resumé of the situation in a non-interacting environment. The original Anderson model is written

$$
H=H_{o}+H_{v}+H_{d}
$$

where

$$
H_{o}=\sum_{\mathbf{k} \sigma} \epsilon_{\mathbf{k}} c^{\dagger} \mathbf{k} \sigma c_{\mathbf{k} \sigma}
$$

describes a sea of conduction electrons,

$$
H_{d}=E_{d} d_{\sigma}^{\dagger} d_{\sigma}+U n_{d \uparrow} n_{d \downarrow}, \quad\left(n_{d \sigma}=d_{\sigma}^{\dagger} d_{\sigma}\right),
$$

is the Hamiltonian for a localized d-state, with an on-site Coulomb interaction of strength $U$, and

$$
H_{v}=V \sum_{\mathbf{k} \sigma}\left[\Phi_{d \mathbf{k}} c^{\dagger} \mathbf{k} \sigma d_{\sigma}+\text { H.c. }\right] .
$$

describes the hybridization between the continuum and the localized atomic orbital. The matrix element 


$$
V \phi_{d \mathbf{k}}=\int d \mathbf{x} e^{i \mathbf{k} \cdot \mathbf{x}} V(\mathbf{x}) \phi_{d}(\mathbf{x})
$$

is the overlap of the local orbital with the surrounding conduction electron orbitals. An important point to note is that the local atomic orbital only hybridizes with a single Wannier state with a particular local symmetry. For a transition metal system, $\phi_{d \mathbf{k}}$ has d- symmetry, in a heavy fermion system this matrix element has f-symmetry. The single-channel nature of the model becomes clear in a tight-binding representation, for if $c^{\dagger}{ }_{j \sigma}=\sum_{\mathbf{k}} c^{\dagger} \mathbf{k} \sigma e^{-i \mathbf{k} \cdot \mathbf{x}_{\mathbf{j}}}$ creates an electron at site $\mathbf{j}$, then $\Phi_{d \mathbf{k}}=\sum_{j} \Phi_{d}\left(\mathbf{x}_{\mathbf{j}}\right) e^{-i \mathbf{k} \cdot \mathbf{x}_{\mathbf{j}}}$ is clearly the form-factor of a Wannier state of nearby atomic orbitals so that $\psi_{d \sigma}^{\dagger}=\sum_{j} \Phi_{d}\left(\mathbf{x}_{\mathbf{j}}\right) c_{j \sigma}^{\dagger}$ creates an electron at this state. In this basis the hybridization can be written

$$
H_{v}=V \sum_{\sigma}\left[d^{\dagger}{ }_{\sigma} \psi_{d \sigma}+\text { H.c. }\right] \text {. }
$$

A large Coulomb interaction $U$ suppresses charge fluctuations on the impurity site, causing local moment formation in the "d-orbital" I In this situation, virtual charge fluctuations induce an anti-ferromagnetic interaction between the local moment and the surrounding conduction sea and the Anderson model can be further reduced by means of a Schrieffer-Wolff 8 transformation which integrates out these fluctuations to yield an effective Kondo model

$$
H=H_{o}+H_{I}
$$

where

$$
H_{I}=J \mathbf{S} \cdot \psi^{\dagger}{ }_{d} \boldsymbol{\sigma} \psi_{d}
$$

describes the residual anti-ferromagnetic interaction between the spin of the local moment $\mathbf{S}=\frac{1}{2} d^{\dagger} \boldsymbol{\sigma} d\left(n_{d}=1\right)$ and the electron spin-density and

$$
J=\left(\frac{V^{2}}{U+E_{d}}\right)+\left(\frac{V^{2}}{-E_{d}}\right)
$$

where $E_{d}$ is taken to be negative. The two terms in this expression are the perturbations to the energy resulting from virtual charge fluctuations $d^{1}+e^{-} \rightleftharpoons d^{2}$ and $d^{1} \rightleftharpoons$ $d^{0}+e^{-}$into the $d^{2}$ and $d^{0}$ configurations respectively. Once again, the local moment only interacts with a single Wannier orbital.

In momentum space the Kondo interaction can be written

$$
H_{I}=\sum_{\mathbf{k}, \mathbf{k}^{\prime}} J_{\mathbf{k}, \mathbf{k}^{\prime}} c^{\dagger} \mathbf{k}_{\mathbf{k}} \boldsymbol{\sigma} c_{\mathbf{k}^{\prime}} \cdot \mathbf{S}
$$

where

$$
J_{\mathbf{k}, \mathbf{k}^{\prime}}=J \Phi_{d \mathbf{k}} \Phi_{d \mathbf{k}^{\prime}}^{*},
$$

involves a single Wannier state. In a site basis, the Kondo interaction becomes

$$
H_{I}=\sum_{l, l^{\prime}} J_{l, l^{\prime}} c^{\dagger}{ }_{l} \boldsymbol{\sigma} c_{l^{\prime}} \cdot \mathbf{S}
$$

where $J_{l, l^{\prime}}=J \Phi_{d}\left(\mathbf{x}_{\mathbf{l}}\right) \Phi_{d}^{*}\left(\mathbf{x}_{\mathbf{l}^{\prime}}\right)$. The non-locality of the exchange means that an electron at a neighboring orbital can exchange spin with the local moment at the same time as hopping to one of the other neighboring orbitals. These are the processes which couple spin and charge fluctuations together.

\section{EFFECT OF INTERACTIONS IN THE ENVIRONMENT}

Now let us discuss how the spin exchange between the local moment and its environment is modified when the surrounding environment becomes interacting. Suppose we introduce a weak spin-spin interaction into the conduction sea, writing

$$
H=H_{o}+H_{I}+\sum_{\mathbf{q}} I(\mathbf{q}) \boldsymbol{\sigma}_{-\mathbf{q}} \cdot \boldsymbol{\sigma}_{\mathbf{q}}
$$

where $\boldsymbol{\sigma}_{\mathbf{q}}=\sum_{\mathbf{k}} \mathbf{c}_{\mathbf{k}-\mathbf{q}}^{\dagger} \boldsymbol{\sigma} \mathbf{c}_{\mathbf{k}}$ is the conduction electron spin-density at momentum $\mathbf{q}$ and $I(\mathbf{q})$ defines the strength of spin-spin interactions at this wavevector. To leading order $O(I)$, there is a vertex correction to the Kondo interaction, as shown in Fig 11. Written out explicitly, this gives

$$
J_{\mathbf{k}, \mathbf{k}^{\prime}}=J_{\mathbf{k}, \mathbf{k}^{\prime}}^{(o)}+J \chi_{d}\left(\mathbf{k}-\mathbf{k}^{\prime}\right) I\left(\mathbf{k}-\mathbf{k}^{\prime}\right)
$$

where

$$
\chi_{d}(\mathbf{q})=2 \sum_{\mathbf{k}} \frac{f\left(\epsilon_{\mathbf{k}-\mathbf{q}}\right)-f\left(\epsilon_{\mathbf{k}}\right)}{\epsilon_{\mathbf{k}}-\epsilon_{\mathbf{k}-\mathbf{q}}} \Phi_{d \mathbf{k}-\mathbf{q}}^{*} \Phi_{d \mathbf{k}},
$$

is the spin-susceptibility of the d-state to a magnetic field at wave-vector $\mathbf{q}$.

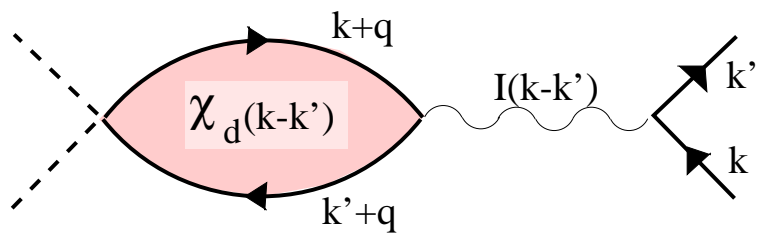

FIG. 1. Vertex correction to Kondo interaction.

By expanding the Kondo coupling in terms of a complete set of orthogonal Wannier states $\left\{\Phi_{\lambda \mathbf{k}}\right\}$ with crystal field symmetry $\lambda, J_{\mathbf{k}, \mathbf{k}^{\prime}}=\sum_{\lambda} J_{\lambda} \Phi_{\lambda \mathbf{k}} \Phi_{\lambda \mathbf{k}^{\prime}}^{*}$, we see that

$$
J_{\lambda}=J \delta_{d \lambda}+J \sum_{\mathbf{k}, \mathbf{k}^{\prime}} \chi_{d}\left(\mathbf{k}-\mathbf{k}^{\prime}\right) I\left(\mathbf{k}-\mathbf{k}^{\prime}\right) \Phi_{\lambda \mathbf{k}}^{*} \Phi_{\lambda \mathbf{k}^{\prime}} .
$$

now contains components in new symmetry channels $\lambda \neq$ $d$. 
To follow how these effects grow with the strength of interaction, we now repeat the analysis in the strong coupling limit, carrying out a Schrieffer-Wolff transformation in the presence of a strongly interacting environment. To be specific, consider a two-dimensional tight-binding model of conduction electrons with a local moment located in the center of a single square plaquet at the origin (Fig. 2). If the onsite Coulomb interaction between the electrons on the lattice is much larger than the bandwidth, the motion of the electrons is described by an infinite U Hubbard modell

$$
H_{o}=\sum_{l, l^{\prime}, \sigma}\left[t_{l l^{\prime}}-\mu \delta_{l l^{\prime}}\right] \mathrm{X}_{l \sigma}^{\dagger} \mathrm{X}_{l^{\prime} \sigma}
$$

where $\mathrm{X}_{j \sigma}=c_{j \sigma}\left(1-n_{j-\sigma}\right)$ is a Hubbard operator 1 and $t_{l l^{\prime}}=-t$ for nearest neighbors, but is zero otherwise. Suppose that the localized state has a d-symmetry, so that

$$
H=H_{o}+H_{v}+H_{d}
$$

where

$$
H_{v}=V \sum_{l \sigma}\left[\Phi_{d}\left(x_{l}\right) d_{\sigma}^{\dagger} \mathrm{X}_{l \sigma}+\mathrm{H} . \mathrm{c}\right]
$$

and

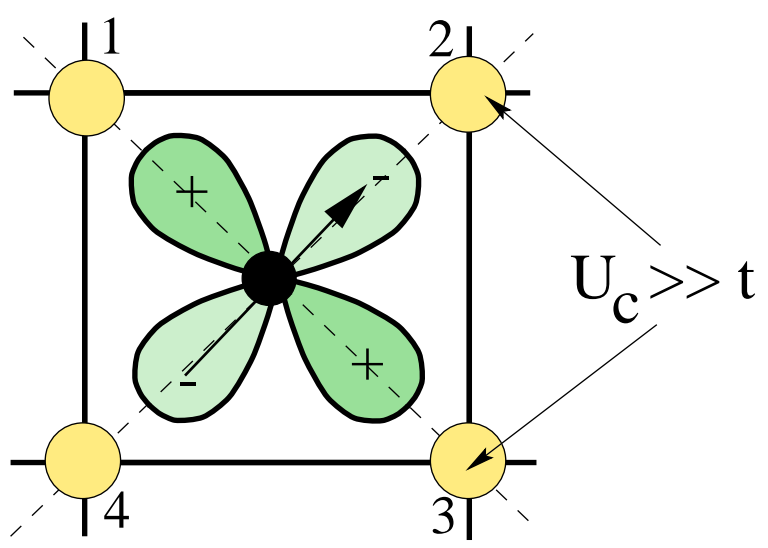

FIG. 2. Magnetic moment in an interacting environment. Localized electron at center of plaquet hybridizes in the $d_{x y}$-channel with nearby atoms. The onsite interaction at each atomic site $U_{c}$ is taken to be far larger than the electron band-width $t$.

With the configuration shown in (Fig 2), it is the $d_{x y}$ orbital of the local moment which hybridizes strongly with the nearby atoms. The value of the $d_{x y}$ Wannier state at the four nearest-neighbor sites labeled sequentially around the local moment (Fig 2) is

$$
\Phi_{\lambda}\left[\mathbf{R}_{i}\right]=\frac{1}{2}(1,-1,1,-1)
$$

where $\Phi(\mathbf{x})=0$ for more distant neighbors. In the limit where $\left|E_{d}\right|$ and $U+E_{d}$ are much larger than both $t$ and $V$, only virtual charge fluctuations take place at the localized moment. We may integrate these fluctuations out by carrying out a Schrieffer Wolff transformation $H \longrightarrow H^{*}=e^{i S} H e^{-i S}$ where $S$ is chosen to eliminate the hybridization term, $i\left[S, H_{o}\right]=-H_{v}$. This yields

$$
H^{*}=H_{o}+H_{I}
$$

where

$$
H_{I}=\left\{\frac{V^{2}}{E_{d}} A A^{\dagger}-\frac{V^{2}}{U+E_{d}} A^{\dagger} A\right\},
$$

where

$$
A=\sum_{l} \Phi_{d}\left(x_{l}\right) d_{\sigma}^{\dagger} \mathrm{X}_{l \sigma}
$$

Re-ordering the operators, we find that

$$
H_{I}=J\left(\mathbf{S} \cdot \Psi^{\dagger}{ }_{d} \boldsymbol{\sigma} \Psi_{d}\right)-K\left(\Psi^{\dagger}{ }_{d} \Psi_{d}\right)
$$

where $J$ is given by (9),

$$
\Psi_{d \sigma}=\sum_{l} \Phi_{d}\left(\mathbf{x}_{\mathbf{l}}\right) \mathrm{X}_{l \sigma},
$$

and

$$
K=\left(\frac{V^{2}}{U+E_{d}}\right)+\left(\frac{V^{2}}{E_{d}}\right) .
$$

For simplicity, we chose the symmetric case, where $U+$ $E_{d}=-E_{d}$ so $K=0$ and potential scattering vanishes. In this case the interaction between the local moment and its environment takes the form

$$
H_{I}=\sum_{l, l^{\prime}} J_{l, l^{\prime}} \mathbf{S} \cdot \mathrm{X}^{\dagger}{ }_{l} \sigma \mathrm{X}_{l^{\prime}},
$$

where $J_{l, l^{\prime}}=J / 4$ for all sites around the spin. We see that the net effect of the strong interactions in the environment is to replace the conduction electron operators by Hubbard operators

$$
c_{j \sigma} \longrightarrow c_{j \sigma}\left(1-n_{j-\sigma}\right)=\mathrm{X}_{j \sigma}
$$

We now examine the consequences of this replacement.

We may divide the Kondo interaction into a one-site and two-site component, writing

$$
J_{l, l^{\prime}}=(J / 4)\left[\delta_{l l^{\prime}}+\left(1-\delta_{l, l^{\prime}}\right)\right]
$$

These two terms are the loose analog of forward, and backward scattering in one dimension. The site diagonal terms do not involve charge fluctuations and these are unaffected by the presence of interactions. By contrast, processes where the electron exchanges spin and hops 
from site to site are suppressed by the Coulomb interactions in the conduction sea: these processes are completely eliminated in the limit where there is one electron per site.

We may make a crude estimation of the effect of the Hubbard operators by making a Gutzwiller approximation:

$$
\mathrm{X}^{\dagger}{ }_{j} \boldsymbol{\sigma} \mathrm{X}_{l} \longrightarrow c^{\dagger}{ }_{j} \boldsymbol{\sigma} c_{l} \times\left\{\begin{array}{cc}
1, & (j=l) \\
(1-x), & (j \neq l)
\end{array}\right.
$$

where $x$ is the concentration of electrons. This approximation yields the right physics for $x \sim 0$ and in the limit $x \rightarrow 1$. It follows that

$$
\begin{aligned}
H_{I} & =\sum_{l, l^{\prime}} J_{l l^{\prime}} \mathbf{S} \cdot c^{\dagger}{ }_{l} \boldsymbol{\sigma} c_{l^{\prime}}, \\
J_{l, l^{\prime}} & =\frac{J}{4}\left[(1-x)+x \delta_{l l^{\prime}}\right]
\end{aligned}
$$

The first term in $J_{l, l^{\prime}}$ describes spin exchange in the original single channel. The second term is site-diagonal and therefore involves a sum over new spin exchange channels. For this lattice there are four orthogonal Wannier states $\Phi_{\lambda}, \lambda=(1,4)$ which overlap with the nearest neigbor atoms. The value of the Wannier state at the four sites labeled sequentially around the local moment is then

$$
\Phi_{\lambda}\left(\mathbf{R}_{i}\right)=\frac{1}{2}\left(1,-i^{\lambda},(-1)^{\lambda},-(-i)^{\lambda}\right)
$$

where we identify $\Phi_{0} \equiv \Phi_{d}$, with the primary d-channel. $\lambda=1$ and $\lambda=3$ correspond to p-channels, whereas $\lambda=3$ describes the extended s-channel. If we expand $J_{l, l^{\prime}}$ in this basis, writing $J_{\lambda}=\sum_{l, l^{\prime}} J_{l, l^{\prime}} \Phi_{\lambda}^{*}\left(\mathbf{x}_{\mathbf{l}}\right) \Phi_{\lambda}\left(\mathbf{x}_{\mathbf{1}^{\prime}}\right)$, we find that

$$
J_{\lambda} / J=\left\{\begin{array}{cl}
1-\frac{3 x}{4}, & \lambda=0, \quad \text { Primary ch. } \\
\frac{x}{4}, \quad \lambda=1,2,3, & \text { Secondary ch. }
\end{array}\right.
$$

so that interactions induce spin exchange in three new channels: two p- and one extended s-channel, each with scattering amplitude $J x / 4$. Schematically

$$
\mathrm{d} \text {-channel } \stackrel{\text { interactions }}{\longrightarrow} \mathrm{d}, \mathrm{p}, \text { s-channel }
$$

We may compactly represent the spin-exchange by replacing $J_{\mathbf{k}, \mathbf{k}^{\prime}}$ in (11) by

$$
J_{\mathbf{k}, \mathbf{k}^{\prime}}=\sum_{\lambda=0,3} J_{\lambda} \Phi_{\lambda \mathbf{k}} \Phi_{\lambda \mathbf{k}^{\prime}}^{*}
$$

Remarkably, the strength of the scattering in the other channels is broadly comparable with that in the primary channel, and in the extreme limit of one electron per site $(x=1)$, the amplitude to scatter becomes equal in each channel. In this special limit, all spin-hop processes have been suppressed, and the Kondo interaction becomes four individual Heisenberg spin couplings to each neighboring atom. This means that in the vicinity of a Mott transition, a local moment will behave as a multi-channel Kondo model.

\section{CHANNEL SYMMETRY AND IMPLICATIONS FOR THE KONDO LATTICE}

Physical realizations of a Kondo lattice will always involve electron interactions in the conduction sea. From the arguments we have just developed, we expect these interactions to induce a Kondo coupling in new symmetry channels. Predominantly f-channel heavy fermion systems are expected to develop weaker spin-exchange couplings to the d, p and s-channels. Likewise, d-channel transition metal systems will develop weaker Kondo coupling to the $\mathrm{p}$ and s-channels.

At first sight, these weaker secondary couplings might be thought to be irrelevant as they are for example, in a single impurity model12 14 . For an impurity magnetic ion, the Kondo effect develops exclusively in the strongest screening channel. However, Kondo impurity models have a special local symmetry which preserves the channel quantum number of scattered electrons. By contrast, an electron travelling in a Kondo lattice can change symmetry channels as it moves from one spin site to another, so that channel quantum number is not conserved. This has a profound influence on the Kondo lattice, for it means that the the subspace of Kondo singlets in one channel is no longer orthogonal to the subspace of Kondo singlets in other channels. Thus the development of Kondo effect in one channel no longer excludes the possibility of a Kondo effect developing coherently in the other channels.

To illustrate this point we shall consider a two-channel Kondo lattice in the strong coupling limit, where the band-width is set to zero, so

$$
\begin{aligned}
H & =H^{(1)}+H^{(2)} \\
H^{(\lambda)} & =\left(J_{\lambda} / N_{s}\right) \sum_{\mathbf{k}, \mathbf{k}^{\prime}, j} \Phi_{\lambda \mathbf{k}} \Phi_{\lambda \mathbf{k}^{\prime}}^{*} c^{\dagger}{ }_{\lambda \mathbf{k}} \boldsymbol{\sigma} c_{\lambda \mathbf{k}^{\prime}} \cdot \mathbf{S}_{j} e^{i\left(\mathbf{k}^{\prime}-\mathbf{k}\right) . \mathbf{R}_{j}},
\end{aligned}
$$

where $\sum_{\mathbf{k}} \Phi_{1 \mathbf{k}} \Phi_{2 \mathbf{k}}^{*}=0$ defines the orthogonality between the channels and $N_{s}$ is the number of sites in the lattice.

Let us now contrast the effect of $H^{(2)}$ in a single impurity model, with its effect in a lattice. (Fig. 3) Suppose $J_{1}>>J_{2}$, so that the low-energy physics is determined by the projection of $H$ into the space of Kondo singlets in channel one. First consider an impurity model. For $J^{(2)}=0$, the ground-state is a Kondo singlet formed between the local moment, and an electron in channel one

$$
|\phi\rangle=\frac{1}{2}\left[\psi_{1 \uparrow}^{\dagger} d_{\downarrow]}^{\dagger}-\psi_{1 \downarrow}^{\dagger} d_{\uparrow}^{\dagger}\right]|0\rangle
$$


where $\psi_{1 \sigma}^{\dagger}=N^{-1 / 2} \sum_{\mathbf{k}} \Phi_{1 \mathbf{k}} c^{\dagger} \mathbf{k} \sigma$, and we have represented $\mathbf{S}=d^{\dagger}\left(\frac{\boldsymbol{\sigma}}{2}\right) d$. Now $H^{(2)}$ flips the spin of the local moment without affecting the spin of the electron it is bound to. To see this, note that $H^{(2)}=J_{2} \mathbf{S} \cdot \psi_{\mathbf{2}}^{\dagger} \boldsymbol{\sigma} \psi_{\mathbf{2}}$ where $\psi_{2}^{\dagger}=N^{-1 / 2} \sum_{\mathbf{k}} \Phi_{2 \mathbf{k}} c^{\dagger} \mathbf{k}$. Orthogonality of the scattering channels guarantees that $\left\{\psi_{1 \sigma}, \psi_{2 \sigma^{\prime}}^{\dagger}\right\}=0$, i.e $\psi_{2}$ has no overlap with the bound-electron in channel one. This means that when we project $H^{(2)}$ into the low-energy subspace,

$$
H^{(2)} \rightarrow\langle\phi|\mathbf{S}| \phi\rangle \cdot \psi_{2}^{\dagger} \boldsymbol{\sigma} \psi_{2}=0
$$

because there are no matrix elements of the spin operator $\mathbf{S}$ in the singlet subspace.



FIG. 3. Contrasting the strong-coupling limit of a single impurity and lattice model with a weak second-channel coupling. In the impurity model, there is no matrix element of $H^{(2)}$ in the low-energy subspace. In the lattice, where channel number is not conserved, the matrix element of $H^{(2)}$ in the low-energy subspace is finite, and gives rise to interactions amongst the mobile Zhang-Rice singlets.

By contrast, in the lattice where channel conservation is lost, $H^{(2)}$ does act on the electrons bound into Kondo singlets, so that there are finite matrix elements of $H^{(2)}$ in the low-lying singlet subspace of channel one. If $|\alpha\rangle$ and $|\beta\rangle$ are states in this low-lying subspace, this means

$$
\left\langle\alpha\left|H^{(2)}\right| \beta\right\rangle=\left\{\begin{array}{cc}
0, & \text { impurity } \\
O\left(J_{2}\right), & \text { lattice }
\end{array}\right.
$$

This marks a qualitative difference between the impurity and lattice models. It means that we can no longer tacitly assume that in the lattice second-channel couplings are an irrelevant perturbation.

We now calculate the form of these additional terms in the lattice. We follow the method developed by Zhang and Rice for reducing a two-band madel of the cuprate perovskites to a one-band t-J model3. The Zhang-Rice reduction to a single band was carried out on a model with spin-exchange in a single $\left(d_{x^{2}-y^{2}}\right)$ channel. We now examine how this analysis changes when a weak additional spin exchange channel is introduced. We first construct a set of orthogonal Zhang-Rice singlet operators for channel one. An electron in the Wannier state with the symmetry of channel one is created at site $j$ by the operator

$$
p_{j \sigma}^{\dagger}=\frac{1}{\sqrt{N}} \sum_{\mathbf{k}} \frac{\Phi_{1 \mathbf{k}}}{\left|\Phi_{1 \mathbf{k}}\right|} e^{i \mathbf{k} \cdot \mathbf{R}_{\mathbf{j}}} c_{\mathbf{k} \sigma}^{\dagger}
$$

We can write both $H^{(1)}$ and $H^{(2)}$ in this basis as follows

$$
H^{(\lambda)}=\frac{J_{\lambda}}{N_{s}} \sum_{\mathbf{k}, \mathbf{k}^{\prime}, j} \tilde{\Phi}_{\lambda \mathbf{k}} \tilde{\Phi}_{\lambda \mathbf{k}^{\prime}}^{*} p^{\dagger}{ }_{\mathbf{k}} \sigma p_{\mathbf{k}^{\prime}} \cdot \mathbf{S}_{j} e^{i\left(\mathbf{k}^{\prime}-\mathbf{k}\right) \cdot \mathbf{R}_{j}}
$$

where

$$
\tilde{\Phi}_{\lambda \mathbf{k}}=\left|\Phi_{\lambda \mathbf{k}}\right|\left[\frac{\Phi_{1 \mathbf{k}}^{*} \Phi_{\lambda \mathbf{k}}}{\left|\Phi_{1 \mathbf{k}}\right|\left|\Phi_{\lambda \mathbf{k}}\right|}\right]
$$

Our ability to write $H^{(2)}$ in terms of the Wannier states of channel one is a direct consequence of the absence of channel conservation.

The low-lying basis of Zhang-Rice singlets for $H^{(1)}$ is constructed using the operator

$$
b_{j}^{\dagger}=\frac{1}{\sqrt{2}}\left[p_{j \uparrow}^{\dagger} d_{j \downarrow}^{\dagger}-p_{j \downarrow}^{\dagger} d_{j \uparrow}^{\dagger}\right]
$$

to creat a "Zhang-Rice" singlet in channel one at site $j$. In the low-lying manifold of states, each site is either occupied by a Zhang-Rice singlet, or an isolated d-spin. The vacuum corresponds to a singlet at every site

$$
|\phi\rangle=\prod_{j} b_{j}^{\dagger}|0\rangle
$$

and a general state is formed by acting on this state with the Hubbard operator $\mathrm{X}_{j \sigma}^{\dagger}=\sqrt{2} d_{j \sigma}^{\dagger} b_{j}$ as follows

$$
\left|\left\{j \sigma_{j}\right\}\right\rangle=\prod_{\left\{j, \sigma_{j}\right\}} \mathrm{X}_{j \sigma_{j}}^{\dagger}|\phi\rangle \text {. }
$$

Within this manifold of states an electron can only be added by the creation of a Zhang-Rice singlet. For states $|\alpha\rangle,|\beta\rangle$ that lie in the low-lying subspace $\left|\left\{j \sigma_{j}\right\}\right\rangle$,

$$
\left\langle\alpha\left|p_{j \sigma}^{\dagger}\right| \beta\right\rangle=\left\langle\alpha\left|\sqrt{2} \sigma b_{j}^{\dagger} d_{j,-\sigma}\right| \beta\right\rangle=\left\langle\alpha\left|\sigma \mathrm{X}_{j-\sigma}\right| \beta\right\rangle
$$

so we may carry out the projection into the low-energy subspace by replacing $p_{j \sigma}^{\dagger} \rightarrow \sigma \mathrm{X}_{j}-\sigma$. The projected form for $H^{(\lambda)}$ is then

$$
H^{(\lambda)}=\frac{J_{\lambda}}{N_{s}} \sum_{l, l^{\prime}, j} \tilde{\Phi}_{\lambda}\left(\mathbf{x}_{l^{\prime} j}\right) \tilde{\Phi}_{\lambda}^{*}\left(\mathbf{x}_{l j}\right) \mathrm{X}^{\dagger} l^{\prime} \boldsymbol{\sigma} \mathrm{X}_{l} \cdot \mathbf{S}_{j}
$$

On the sites where $l=j$ or $l^{\prime}=j$, we can use the identity $\left(\mathbf{S}_{j} \cdot \boldsymbol{\sigma}\right) \mathrm{X}_{j}=-\frac{3}{2} \mathrm{X}_{j}$, to obtain 


$$
\begin{aligned}
H^{(\lambda)} & =\sum_{\mathbf{i}, \mathbf{j}} t_{i j}^{\lambda} \mathrm{X}_{i \sigma}^{\dagger} \mathrm{X}_{j \sigma} \\
& +\frac{J_{\lambda}}{N_{s}} \sum_{l, l^{\prime} \neq j} \tilde{\Phi}_{\lambda}\left(\mathbf{x}_{l^{\prime} j}\right) \tilde{\Phi}_{\lambda}^{*}\left(\mathbf{x}_{l j}\right) \mathrm{X}_{l}^{\dagger} \boldsymbol{\sigma} \mathrm{X}_{l^{\prime}} \cdot \mathbf{S}_{j},
\end{aligned}
$$

where $t_{j l}^{\lambda}=N_{s}^{-1} \sum_{\mathbf{k}} t_{\lambda}(\mathbf{k}) e^{i \mathbf{k} \cdot \mathbf{R}_{\mathbf{j} 1}}$ and

$$
t_{\lambda}(\mathbf{k})=-3 J_{\lambda} \tilde{\Phi}_{\lambda}(0) \operatorname{Re}\left[\tilde{\Phi}_{\lambda \mathbf{k}}\right]
$$

The first term in (47) describes the motion of the ZhangRice holes. In general, $\tilde{\Phi}_{2 \mathbf{k}}$ is a function with nodes, so $\tilde{\Phi}_{2}(0)$ vanishes, and $H^{(2)}$ contributes solely to an anisotropic interaction amongst the holes.

$$
H^{(2)}=J_{2} \sum_{l, l^{\prime}, j} \tilde{\Phi}_{2}\left(\mathbf{x}_{l^{\prime} j}\right) \tilde{\Phi}_{2}^{*}\left(\mathbf{x}_{l j}\right) \mathrm{X}_{l}^{\dagger} \boldsymbol{\sigma} \mathrm{X}_{l^{\prime}} \cdot \mathbf{S}_{j},
$$

The symmetry of this term is governed by the product of form-factors $\tilde{\Phi}_{2 \mathbf{k}} \propto \Phi_{2 \mathbf{k}} \Phi_{1 \mathbf{k}}^{*}$, a function that has to contain nodes, because of the orthogonality of form-factors $\left(\sum_{\mathbf{k}} \Phi_{2 \mathbf{k}} \Phi_{1 \mathbf{k}}^{*}=0\right)$. In the primary channel, the corresponding interaction term has an isotropic "extended-s" symmetry. This term is numerically small and is generally neglected as an irrelevent perturbation to the infinite (s-wave) onsite repulsion between holes. The final form for the effective Hamiltonian is

$H=t \sum_{(i, j)} \mathrm{X}_{i}^{\dagger} \mathrm{X}_{j}+\frac{J_{2}}{N_{s}} \sum_{j, \mathbf{a}, \mathbf{a}^{\prime}} \tilde{\Phi}_{2}(\mathbf{a}) \tilde{\Phi}_{2}^{*}\left(\mathbf{a}^{\prime}\right) \mathrm{X}_{j+\mathbf{a}}^{\dagger} \sigma \mathrm{X}_{j+\mathbf{a}^{\prime}} \cdot \mathbf{S}_{j}$

where, we have neglected all but the nearest neighbor coefficients, so that $(i, j)$ represent nearest neigbors, $\mathbf{a}$ is a vector linking nearest neigbors, $t=-3 \tilde{\Phi}_{1}(0) \tilde{\Phi}_{1}(\mathbf{a}) J_{1}$. The second term shows that spin-exchange processes in channel two survive the projection into the subspace of singlets for channel one. For this reason, we can no longer expect singlet formation in one channel to pre-empt a Kondo effect in the second, weaker channel.

One of the interesting possibilities that this presents us with, is the possibility that Kondo spin-exchange in the second-channel can generate pairing. If we consider a pair of Zhang-Rice holes, then the matrix elements between the two states produced by $H^{(2)}$ is given by

$$
\left\langle\mathbf{k} \uparrow,-\mathbf{k} \downarrow\left|H^{(2)}\right| \mathbf{k}^{\prime} \uparrow,-\mathbf{k}^{\prime} \downarrow\right\rangle \propto-J_{2} \tilde{\Phi}_{2 \mathbf{k}} \tilde{\Phi}_{2 \mathbf{k}^{\prime}}^{*} .
$$

In the original Zhang-Rice problem, the primary spinexchange channel has $d_{x^{2}-y^{2}}$ symmetry. The projected form factor for the primary $d_{x^{2}-y^{2}}$ spin-exchange channel is

$$
\tilde{\Phi}_{1 \mathbf{k}}=\left(1+\frac{1}{2}\left[\cos \left(k_{x}\right)+\cos \left(k_{y}\right)\right]\right)^{\frac{1}{2}}
$$

We expect there to also be spin-exchange terms of strength $J_{2} \sim(\delta / 8) J_{1}$, where $\delta$ is the doping, in the $\mathrm{p}$ and extended s-channels 15 Of these, the most interesting component is that with extended s-symmetry, for in this case $\tilde{\Phi}_{2 \mathbf{k}}$ has the product symmetry $s \otimes d_{x^{2}-y^{2}}=d_{x^{2}-y^{2}}$, which has even parity and can support singlet pairing. A careful calculation gives

$$
\tilde{\Phi}_{2 \mathbf{k}}=\left[\cos \left(k_{y}\right)-\cos \left(k_{x}\right)\right] /\left(2 \Phi_{1 \mathbf{k}}\right)
$$

Since $J_{2} / J_{1} \sim(x / 8)$, this is a small, but significant perturbation to the model. Were it to lead to a genuine pair instability, the microscopic description of the state that forms would involve the coherent presence of Zhang-Rice singlets of two distinct symmetries. This is a topic we shall return to in a forthcoming paper.10

\section{CONCLUSION}

This paper has examined the effect of interactions around a local moment. Conventional wisdom assumes that a localized moment scatters electrons in a symmetry channel of the same local symmetry. We have shown that electron interactions cause a local moment to exchange spin with electrons in scattering channels with different local symmetry. Close to a Mott transition these effects are extreme, and the spin-exchange Hamiltonian acquires the symmetry of a multichannel Kondo problem. Finally, we have discussed how these new interaction terms become important in the Kondo lattice, where the absence of a conserved channel index means that second-channel couplings generate important interactions within the the low-energy subspace of Kondo singlets. The possible consequences of these new couplings will be analyzed in a subsequent paper.

We should like to thank the ITP, Santa Barbara, where the early discussions leading to this work took place under the support of NSF grants DMR-92-23217 and PHYS94-07194. Discussions with S. Kivelson on related topics during this period are gratefully acknowledged. Later work was supported in part by the National Science Foundation under Grant DMR 96-14999, the EPSRC, UK and NATO grant CRG. 940040 during a sabbatical stay at Oxford.

* On sabbatical leave from Rutgers University.

${ }^{1}$ P. W. Anderson, Phys. Rev 124, 41, (1961).

2 J. Hubbard, Proc. Roy. Soc A281, 401, (1964).

${ }^{3}$ F. C. Zhang and M. Rice, Phys Rev. B37, 3664, (1988).

${ }^{4}$ C. L. Kane and M. P. A. Fisher, Phys. Rev. Lett. 68, 1220 (1992).

${ }^{5}$ Dung-Hai Lee and John Toner, Phys. Rev. Lett. 69, 3378, (1992).

${ }^{6}$ A. Furusaki and N. Nagaosa PRL 72, 892 (1994).

7 T. Schork and P. Fulde, Phys. Rev. B 50, 1345 (1994).

${ }^{8}$ J. R. Schrieffer and P. Wolff, Phys. Rev. 149, 491, (1966). 
${ }^{9}$ V. J. Emery and S. A. Kivelson, Phys. Rev. B46, 10812 (1992).

${ }^{10}$ P. Coleman, A. Tsvelik, N. Andrei and H. Y. Kee, condmat/9707002.

${ }^{11}$ O. Zachar, S. A. Kivelson and V. J. Emery, Phys. Rev. Lett. 77, 1342 (1996); P. Coleman, A. Georges and A. M. Tsvelik, J. Phys: Cond. Matt 9, 345 (1997).

12 P. Nozières and A. Blandin, J. Phys. (Paris) 41, 193 (1980).

${ }^{13}$ N. Andrei and A. Jerez, Phys. Rev. Lett. 74, 4507 (1995).

${ }^{14}$ D. L. Cox and A. Zawadowski, "Exotic Kondo Effects in Metals", cond-mat 9704103 (submitted to Rev. Mod. Phys) and references therein.

${ }^{15}$ In the cuprate metals, there are two in-plane oxygen atoms per copper site, so that the doping per oxygen atom is onehalf the overall electron doping, $x=\delta / 2$. From section (III) our estimate of $J_{2}$ is thus $J_{2}=J_{2} \delta / 8$. 